ARTICLE

\title{
Non-equilibrium metal oxides via reconversion chemistry in lithium-ion batteries
}

\author{
Xiao Hua (1) ${ }^{1 凶}$, Phoebe K. Allan², Chen Gong (1) ${ }^{3}$, Philip A. Chater (1) 4, Ella M. Schmidt (1) ${ }^{1}$, Harry S. Geddes ${ }^{1}$, \\ Alex W. Robertson ${ }^{3}$, Peter G. Bruce $\mathbb{1}^{3}{ }^{3} \&$ Andrew L. Goodwin (10 ${ }^{1}$
}

Binary metal oxides are attractive anode materials for lithium-ion batteries. Despite sustained effort into nanomaterials synthesis and understanding the initial discharge mechanism, the fundamental chemistry underpinning the charge and subsequent cycles-thus the reversible capacity-remains poorly understood. Here, we use in operando X-ray pair distribution function analysis combining with our recently developed analytical approach employing Metropolis Monte Carlo simulations and non-negative matrix factorisation to study the charge reaction thermodynamics of a series of Fe- and Mn-oxides. As opposed to the commonly believed conversion chemistry forming rocksalt $\mathrm{FeO}$ and $\mathrm{MnO}$, we reveal the two oxide series topotactically transform into non-native body-centred cubic $\mathrm{FeO}$ and zincblende $\mathrm{MnO}$ via displacement-like reactions whose kinetics are governed by the mobility differences between displaced species. These renewed mechanistic insights suggest avenues for the future design of metal oxide materials as well as new material synthesis routes using electrochemically-assisted methods.

\footnotetext{
${ }^{1}$ Inorganic Chemistry Laboratory, University of Oxford, Oxford OX1 3QR, UK. ${ }^{2}$ School of Chemistry, University of Birmingham, Birmingham B15 2TT, UK. ${ }^{3}$ Department of Materials, University of Oxford, Parks Road, Oxford OX1 3PH, UK. ${ }^{4}$ Diamond Light Source Ltd, Harwell Science and Innovation Campus,

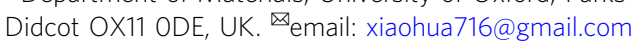


$\mathrm{T}$ he vast majority of electrode materials operate via insertion chemistry ${ }^{1}$. Their performance is restricted by the homogeneity range of their crystal structures to (usually) $1 e^{-}$ transfer (per formula unit) upon cycling, which limits their capacity. The search for next-generation materials with higher capacities is therefore essential to improve battery performance. As an alternative to insertion materials, conversion materials experience a complete reduction of the metal component upon discharge: $(z \cdot n) \mathrm{Li}+\mathrm{M}_{y} X_{z} \leftrightarrow y \mathrm{M}+z \mathrm{Li}_{n} X(X=\mathrm{O}, \mathrm{S}, \mathrm{P}, \mathrm{F}$, etc. $)$. Such a reaction involves multiple- $e^{-}$transfer yielding high theoretical capacities (e.g., $1007 \mathrm{mAh} / \mathrm{g}$ for $\alpha-\mathrm{Fe}_{2} \mathrm{O}_{3}$ c.f. $372 \mathrm{mAh} / \mathrm{g}$ for graphite $)^{2}$ and, thus, these systems have consequently attracted considerable interest. However, the crystal structures of these materials are believed to undergo a complete de- and reconstruction upon cycling-accompanied by significant volume changes that may impair the electrode's mechanical integrityleading to large voltage hysteresis, limited cycle life, poor rate performance and mediocre capacity retention ${ }^{1,2}$.

With the aim of enhancing their performance, much of the relevant research effort has focussed on material nanostructuring to promote efficient reaction with $\mathrm{Li}$. In this respect, first-row transition-metal oxides $\left(M_{x} \mathrm{O}_{y}\right)$-and particularly the $\mathrm{Fe}$ and $\mathrm{Mn}$ series $(M=\mathrm{Fe}, \mathrm{Mn})$ - have become the most studied conversion compounds due to their low cost and straightforward syntheses ${ }^{3}$. As a consequence, we now have a library of $M_{x} \mathrm{O}_{y}$ systems with diverse nanomorphologies ${ }^{4}$. Although these engineered oxides have proven effective in improving rate capability and cyclability, critical issues such as low power and energy efficiency remain key obstacles to commercial application ${ }^{1}$. The development of viable strategies to overcome these issues therefore demands a muchimproved understanding of the underlying reaction mechanisms.

Regardless of the particular $M_{x} \mathrm{O}_{y}$ starting phase, these oxides undergo an electrochemical pulverisation during the conversion processes that leads to the formation of near-amorphous $M$ and $\mathrm{Li}_{2} \mathrm{O}$ at the end of the first discharge ${ }^{5-8}$ (Fig. 1a). This $M-\mathrm{Li}_{2} \mathrm{O}$ mixture subsequently reconverts to an amorphous and/or disordered metal oxide phase (hereafter referred to as $M_{x^{\prime}} \mathrm{O}_{y^{\prime}}$ ) upon charge. Despite lacking long-range order, this $M_{x} \mathrm{O}_{y^{\prime}}$ was found to locally resemble a rocksalt $(r s)$ type $M O$ structure ${ }^{5,7,9}$ in both $\mathrm{Fe}$ and $\mathrm{Mn}$ systems, in turn suggesting their delithiation might follow related pathways. More importantly, the following discharge step seems to mirror the charging process, implying the actual reversible reaction might be independent on the starting $M_{x} \mathrm{O}_{y}$ material. While earlier studies of these systems focussed predominantly on their initial insertion and conversion chemistry, it is critical to understand the reconversion mechanism and subsequent cycling steps, considering that these are the key processes that define the reversible capacity. Given the heterogeneous and nanoscopic nature of the (re)conversion process ${ }^{5,10}$, the characterisation used in previous studies primarily relied on conventional techniques such as X-ray diffraction $(\mathrm{XRD})^{5,8}$, transmission electron microscopy (TEM) $)^{9,11,12}$ and X-ray absorption spectroscopy (XAS) $)^{7,8,11}$. Even with many in operando/in situ measurements undertaken, the inability of XRD to determine the short-range structure and the insensitivity of EXAFS to structure beyond the first few coordination shells ${ }^{13}$ means that the structural changes upon charging $M_{x} \mathrm{O}_{y}$ materials remain unestablished.

In this work, we seek to address these main challenges using in operando and in situ (hereafter simplified as in situ) pair distribution function (PDF) via X-ray total scattering, a powerful tool for studying various types of conversion materials ${ }^{14-16}$. For a systematic and robust investigation, we have selected a series of $\mathrm{Fe}_{x} \mathrm{O}_{y}$ and $\mathrm{Mn}_{x} \mathrm{O}_{y}$ compounds and employed an analytical approach including Metropolis Monte Carlo (MMC) simulation ${ }^{17}$ and non-negative matrix factorisation $(\mathrm{NMF})^{18}$. Our results show that these $M_{x} \mathrm{O}_{y}$ systems follow an analogous cycling behaviour within a given $M$ family, but the pathways differ significantly between the $\mathrm{Fe}$ and $\mathrm{Mn}$ series despite their similar electrochemical profiles during reversible cycles. Surprisingly and importantly, although a one-step conversion process from $M$ to $r s-M O$ upon charge was widely accepted in the field, we show that both $\mathrm{Fe}_{x} \mathrm{O}_{y}$ and $\mathrm{Mn}_{x} \mathrm{O}_{y}$ undergo two steps via diffusioncontrolled displacement-like reactions, with $\mathrm{O}^{2-}$ and $\mathrm{Mn}^{2+}$ as

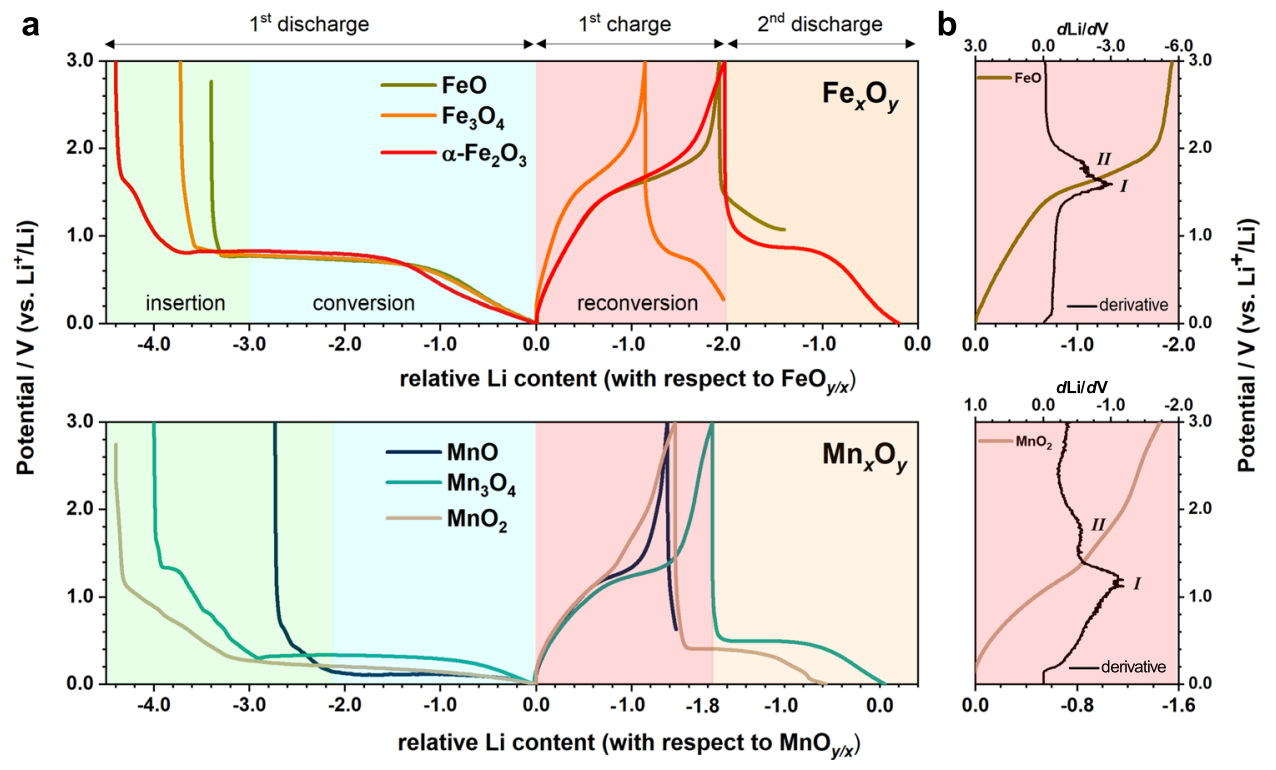

Fig. 1 Electrochemical performance. a The first three cycling steps of $\mathrm{Fe}_{x} \mathrm{O}_{y}$ and $\mathrm{Mn}_{x} \mathrm{O}_{y}$ series from the in situ X-ray total scattering experiments. Distinct processes including insertion, conversion and reconversion in the respective cycling step are labelled. For easy comparison between different $M_{x} \mathrm{O}_{y}$ species, the $x$-axis is against "normalised" and "relative" Li content. "Normalised" means the number of Li is weighted against the number of metal centre ( $x$ ) in $\mathrm{M}_{x} \mathrm{O}_{y}$, i.e., the full theoretical capacity for $\alpha-\mathrm{Fe}_{2} \mathrm{O}_{3}$ corresponds to three $\mathrm{Li}$ per $\mathrm{FeO}_{3 / 2}$. For the "relative" Li content, the value at the end of first discharge is set as a reference against which all other values are adjusted accordingly. b Derivative curves (black) of $\mathrm{FeO}$ and $\mathrm{MnO}_{2}$ plotted in parallel with their corresponding charging profile. Two distinct delithiation steps are labelled using Roman numerals. 
displaced species, respectively, for the $\mathrm{Fe}$ and $\mathrm{Mn}$ systems forming unexpected body-centred cubic $(b c c) \mathrm{FeO}$ and zincblende $(z b) \mathrm{MnO}$. The mechanistic transition from a conversion process to an unexpected topotactic reaction elucidates the fundamental origins of the hysteretic behaviour, offering critical insight into effective material design in the future; on the other hand, it suggests the viability of deriving new MO polymorphs using an electrochemically assisted approach based on battery chemistry. In addition, our study also demonstrates the value of our methodology to investigate battery material systems with complex structures.

\section{Results}

We selected candidate $\mathrm{Fe}_{x} \mathrm{O}_{y}$ and $\mathrm{Mn}_{x} \mathrm{O}_{y}$ materials so as to include the most frequently studied compositional and crystallographic variants. The chosen $\mathrm{Fe}$ species were $\mathrm{FeO}^{19}$ (structure type: rocksalt/space group: $F m \overline{3} m$ ),$\alpha-\mathrm{Fe}_{2} \mathrm{O}_{3}{ }^{10}$ (corundum $/ R \overline{3} c$ ) and $\mathrm{Fe}_{3} \mathrm{O}_{4}{ }^{20}$ (inverse spinel $/ F d \overline{3} m$ ), while the $\mathrm{Mn}_{x} \mathrm{O}_{y}$ series consists of $\mathrm{MnO}^{21}$ (rocksalt/ $F m \overline{3} m$ ), $\mathrm{Mn}_{3} \mathrm{O}_{4}{ }^{22}$ (distorted spinel/
$I 4_{1} /$ amd) and $\beta-\mathrm{MnO}_{2}{ }^{23}$ (rutile/P4 $/ \mathrm{mnm}$ ); the purity of these phases was confirmed by PDF refinement (Supplementary Fig. 1a). Note that these crystal structures are based on classic oxygen-packing frameworks, i.e., cubic-close packing (ccp) in $r s-$ spinel-related structure, hexagonal-close packing $(h c p)$ in hexagonal corundum and tetragonal-close packing $(t c p)^{24}$ in rutile. We thus expect any mechanistic similarity shared within or between the Fe and $\mathrm{Mn}$ families to reflect a general trend for a wider range of $\mathrm{Fe}$ - and $\mathrm{Mn}$-oxide materials.

Electrochemistry. Lithiation of $M_{x} \mathrm{O}_{y}$ begins with an insertion step (in some materials) at a higher voltage than the conversion process (c. 0.8 and $0.3 \mathrm{~V}$ for $\mathrm{Fe}$ and $\mathrm{Mn}$, respectively) and is normally accompanied by electrolyte decomposition and other side reactions ${ }^{15}$, which collectively give rise to the extra capacity seen in the first discharge. The following delithiation (charge) process-which corresponds to the reconversion of $M$ to form the $M_{x^{\prime}} \mathrm{O}_{y^{\prime}}$ phase-exhibits a maximum capacity of about $2 \mathrm{Li}$ (per $M$ ) associated with $2 e^{-}$transfer (per $M$ ) regardless of the number

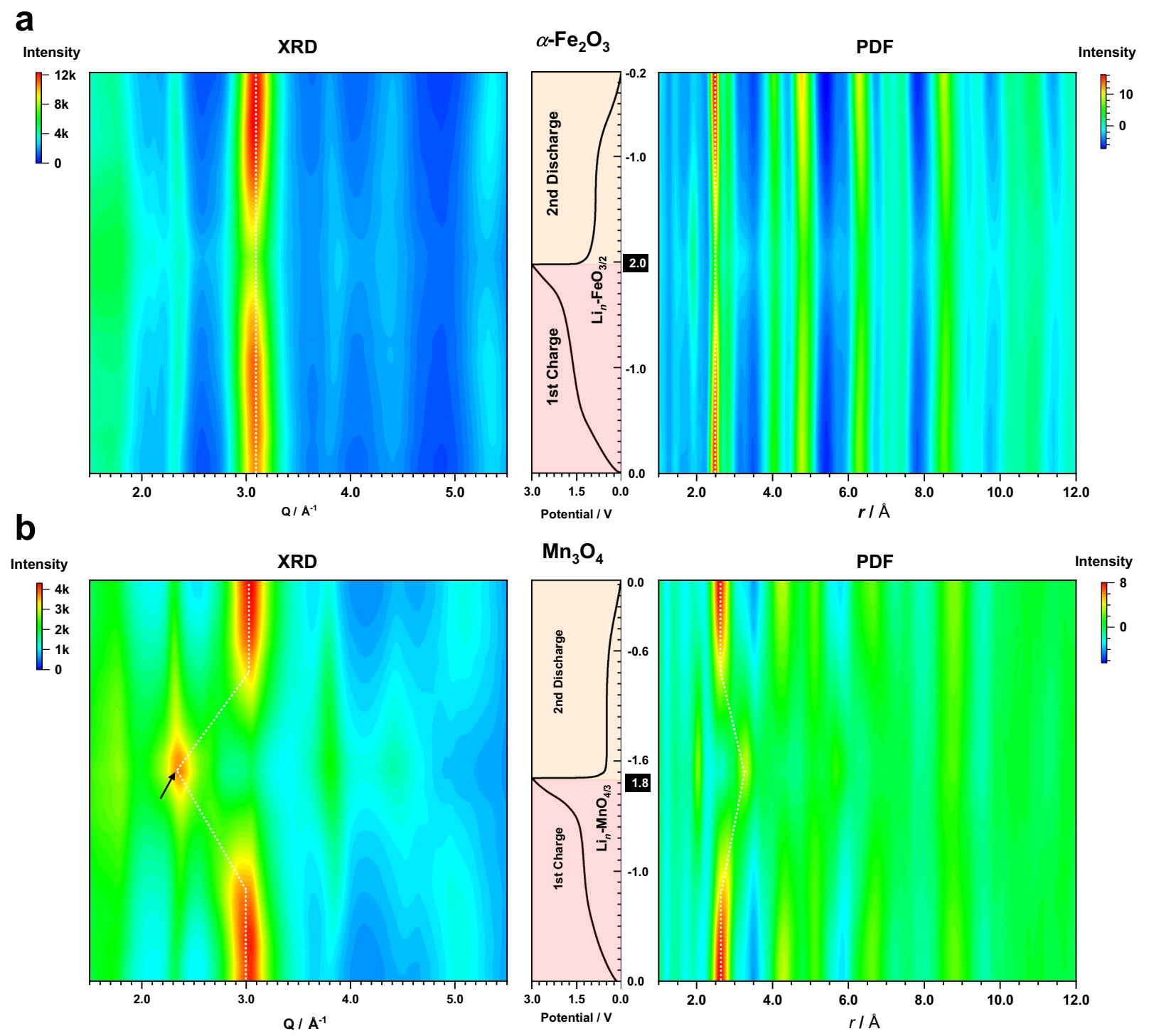

Fig. 2 PDF and XRD patterns. Contour plot of the XRD (left) and PDF (right) patterns extracted from the in situ X-ray total scattering experiments for a $\alpha$ $\mathrm{Fe}_{2} \mathrm{O}_{3}$ and $\mathbf{b} \mathrm{Mn}_{3} \mathrm{O}_{4}$. Each $y$-slice of the contour plot is aligned with the corresponding "normalised relative Li content" (Fig. 1) in the first charge and second discharge cycles. The "relative Li content" at the end of the first charge is highlighted. Bragg reflection corresponding to $z b-\mathrm{MnO}$ is marked with a black arrow. The position change of the most intense peak in each contour plot is marked using a white dotted line. 
of $e^{-}$involved in the initial discharge (Fig. 1a and Supplementary Fig. 1b). Discounting any side reactions after the first discharge, the removal of $2 e^{-}$per metal centre would result in a full oxidation from $M$ to $M^{2+} \mathrm{O}$ species. Based on a nearly identical capacity between the second discharge and the first charge, we can also infer the reconverted $M O$ is the active and reversible oxide component in the following conversion-reconversion cycles. For a clearer comparison between different $M_{x} \mathrm{O}_{y}$ species, the electrochemistry profiles (Fig. 1a) are plotted against the normalised "relative Li content (per $M$ centre)" and will be simplified as "Li content" hereafter unless otherwise stated. It is also worth noting that although most of the $M_{x} \mathrm{O}_{y}$ only exhibit one charge (pseudo-) plateau, two peaks can be resolved in their derivative curves (Fig. 1b) or cyclic voltammograms $(\mathrm{CV})^{19,23,25-28}$. The two maxima are well separated in $\mathrm{Mn}_{x} \mathrm{O}_{y}$, but are less easily distinguished in $\mathrm{Fe}_{x} \mathrm{O}_{y}$. Nevertheless, their presence suggests a two-step phase transition from $M$ to $M O$, deviating from the commonly-assumed single-step reaction $M+\mathrm{Li}_{2} \mathrm{O} \leftrightarrow r s-M \mathrm{O}+2 \mathrm{Li}$.

Characterisation (X-ray total scattering and TEM). Comparing the scattering patterns and PDFs (Fig. 2 and Supplementary Figs. 2-4) for different species within the same $\mathrm{Fe}_{x} \mathrm{O}_{y}$ or $\mathrm{Mn}_{x} \mathrm{O}_{y}$ series, we find that the peak evolution-i.e., peak shifts, appearance and disappearance of Bragg reflections/atom pairs-shows a single common trend. This implies comparable structure changes between different $\mathrm{Fe}_{x} \mathrm{O}_{y}$ or $\mathrm{Mn}_{x} \mathrm{O}_{y}$ systems despite their significant compositional and crystallographic differences at the beginning of first discharge. In addition, the structural change during the second discharge mirrors that during the first charge in both real and reciprocal space (Fig. 2) and the phase changes in the second charge are identical to that in the first charge (Supplementary Fig. 4b, c). This is consistent with a reversible phase behaviour. Based on the observed mechanistic resemblance within the same $M_{x} \mathrm{O}_{y}$ series, we chose $\alpha-\mathrm{Fe}_{2} \mathrm{O}_{3}$ and $\mathrm{Mn}_{3} \mathrm{O}_{4}$ as model compounds for subsequent studies considering that they are the most explored oxide member within each $M_{x} \mathrm{O}_{y}$ family.

To obtain better knowledge of the starting phase for the $1^{\text {st }}$ charge ("Li 0.0 "), quantitative analysis was performed on the scattering data collected at the corresponding state of charge. As confirmed by PDF refinement (Supplementary Fig. 5) and supported by XRD simulation (Supplementary Figs. 6 and 7), the average particle size for $M$ and $\mathrm{Li}_{2} \mathrm{O}$ phases was $\sim 2$ and $3 \mathrm{~nm}$, respectively. TEM analysis of the Fe sample (Fig. 3a-c) shows sphere-like Fe nanoparticles each a few nanometres in size. There is clear evidence of a well-ordered $b c c$ lattice within nanoparticles, which can be discerned despite severe agglomeration. Upon charging, the scattering pattern evolves differently for the two systems (Fig. 2). While $\alpha-\mathrm{Fe}_{2} \mathrm{O}_{3}$ only shows variations in peak intensities with very subtle changes in peak positions, $\mathrm{Mn}_{3} \mathrm{O}_{4}$ exhibits a distinct 'waxing and waning' effect. This contrast implies substantial mechanistic differences in the reconversion processes between the $\mathrm{Fe}_{x} \mathrm{O}_{y}$ and $\mathrm{Mn}_{x} \mathrm{O}_{y}$ series despite their similar electrochemical profiles.

$\alpha-\mathrm{Fe}_{2} \mathrm{O}_{3}$ (model compound for $\mathrm{Fe}_{x} \mathrm{O}_{y}$ ). An earlier study of $\alpha-\mathrm{Fe}_{2} \mathrm{O}_{3}$ using in situ TEM ${ }^{9,11}$ reported a phase transition from $\alpha$ - $\mathrm{Fe}(\operatorname{Im} \overline{3} m)$ to $\mathrm{FeO}(F m \overline{3} m)$ during the charging process. Should this reaction take place, the Fe lattice will undergo a significant rearrangement from $b c c$ to $f c c$ resulting in a pronounced change of the local ordering (Supplementary Fig. 8). Surprisingly, our in situ PDF data (Fig. 4a) only show subtle peak shifts with slight peak broadening and intensity reduction upon charge, implying the $\alpha$-Fe lattice is largely retained without transformation to the $r s$-FeO. TEM measurements (Fig. 3) of the sample at the end of charge ("Li -2.0 ") further confirm the retention of $b c c$ Fe order in the reconverted $\mathrm{FeO}$ structure (hereafter denoted as $b c c-\mathrm{FeO}$ ). In addition, it is also possible to discern atomic displacements (Fig. 3d and Supplementary Fig. 9) that likely account for the increased peak widths in the XRD pattern (Supplementary Fig. 6a). Remarkably, while most of the PDF atom-pair intensities progressively decrease upon charge, a peak at $1.9 \AA$ continues to grow (Fig. 4a). Atomic distances of $\sim 2 \AA$ are characteristic of the first coordination sphere in $\mathrm{Li}_{n} M_{x} \mathrm{O}_{y}$, and hence correspond to Lior $\mathrm{M}$-centred polyhedral environments. The peak cannot be ascribed to the tetrahedral $\mathrm{Li}-\mathrm{O}$ in the antifluorite $(a f) \mathrm{Li}_{2} \mathrm{O}$ because the concentration of this phase decreases during delithiation. Hence, we assign this peak to a $\mathrm{Fe}-\mathrm{O}$ atom pair, which must correspond to $\mathrm{O}$ inclusion within the $b c c-\mathrm{FeO}$ phase.

The most commonly occupied interstitial site in $b c c$-Fe is the distorted octahedral site, such as is occupied by $X=\mathrm{C}$ or $\mathrm{N}$ in the $\mathrm{Fe}-X$ martensite alloys ${ }^{29-31}$. These sites are located at the centres of the faces and edges of the $b c c$ unit cell (Fig. 4b). There are also 4-coordinated interstitial positions present at the $12 d$ site. Both geometries are distorted compared to their $f c c$-counterparts ${ }^{32}$. An earlier computational study on oxygen diffusivity in $b c c-\mathrm{Fe}$ showed that the octahedrally coordinated oxygen is energetically more favourable than the tetrahedral one (hereafter denoted as
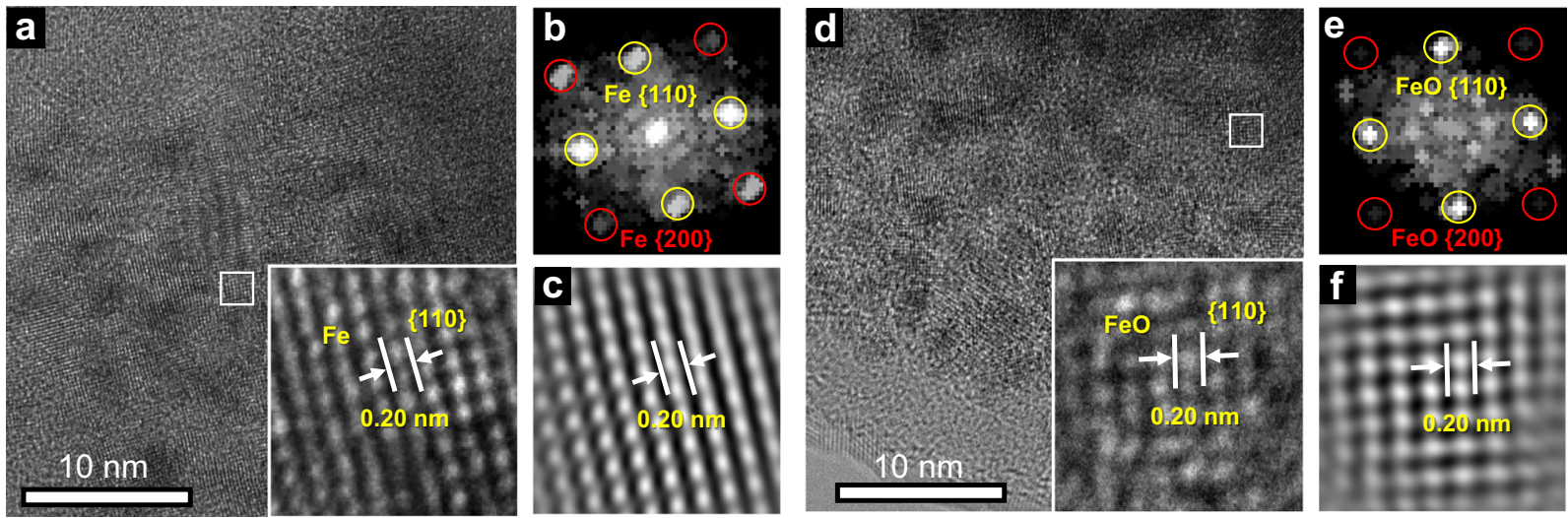

Fig. 3 TEM data for the fully discharged and charged $\boldsymbol{\alpha}-\mathrm{Fe}_{\mathbf{2}} \mathbf{O}_{\mathbf{3}}$. TEM results for the $\alpha-\mathrm{Fe}_{2} \mathrm{O}_{3}$ sample collected at a-c the beginning of charge (" $\mathrm{a} i=0.0^{\prime \prime}$ ) and $\mathbf{d}-\mathbf{f}$ the end of charge ("Li $=-2.0$ "). High-resolution TEM images (a) and (d) show that the bcc-Fe lattice is retained with $\{110\}$ interlayer spacing of about $0.20 \mathrm{~nm}$ (Supplementary Fig. 9) in both structures (scale bar in the bottom left corner indicates $10 \mathrm{~nm}$ ). The preservation of the bcc-Fe ordering is further supported by the indexing in (b) and (e) obtained from fast Fourier transform (FFT) of the highlighted region in (a) and (d). Fourier filtered images (c) and (f) based on FFT exhibit a Fe lattice comparable to the experimental pattern highlighted in (a) and (d). 
a

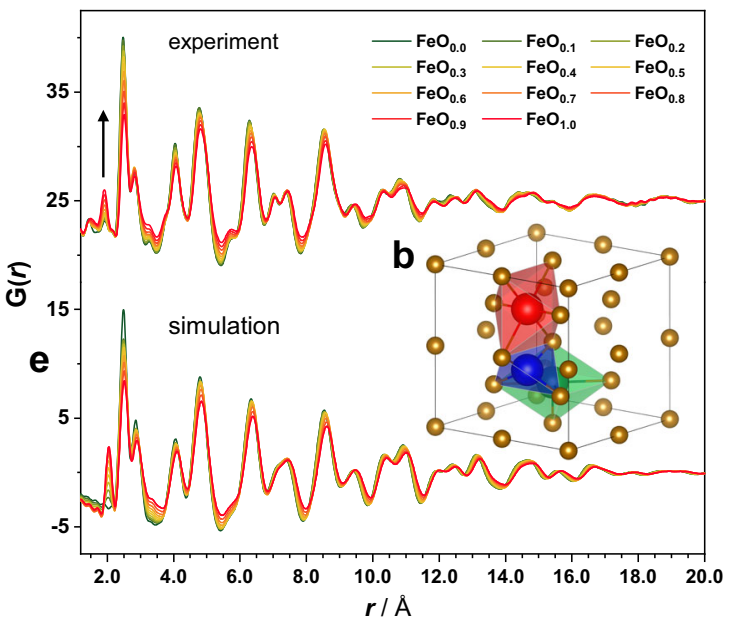

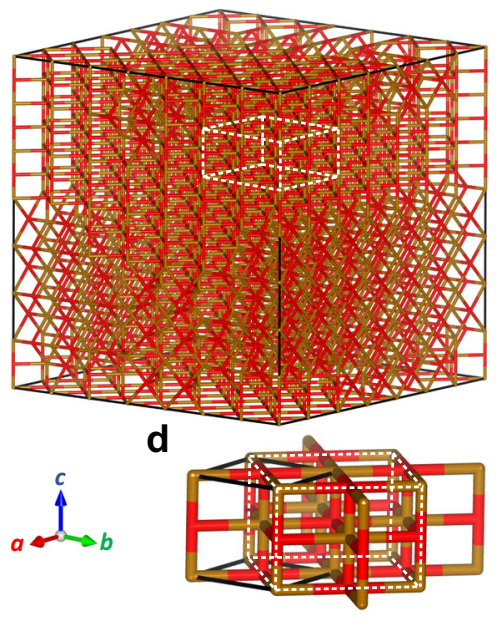

Fig. 4 Structure modelling and PDF simulation for $\mathbf{b c c}_{\mathbf{c}} \mathbf{-} \mathbf{e O}_{\mathbf{x}}$. a Experimental PDF patterns for $\alpha-\mathrm{Fe}_{2} \mathrm{O}_{3}$ extracted from the first charge cycle. Colour gradient from green to red correspond to an increasing concentration of oxygen in $\mathrm{FeO}_{x}$ from $x=0.0$ to 1.0. Black arrow indicates the growth of the $1.9 \AA$ peak upon charge. $\mathbf{b} \alpha$-Fe structure $(2 \times 2 \times 2)$ showing one tet-oxygen (blue) and two oct-oxgyens located on the face (red) and at the edge (green) of the unit cell. c MMC-derived $\mathrm{FeO}_{1.0}$ structure $(7 \times 7 \times 7 \alpha$-Fe unit cell). For a clearer view, the structure is presented using a stick model (Fe: brown; O: red). d A repeating unit taken from an ordered domain highlighted in (c). The black solid frame denotes a bcc-FeO unit with the $14 / \mathrm{mmm}$ symmetry and the

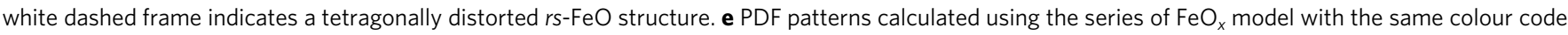
shown in (a).

oct- and tet-O, respectively $)^{33}$. In the absence of any local structural relaxation due to the occupation of interstitial atoms, the six $\mathrm{Fe}-\mathrm{O}$ pairs affiliated with the oct-O should contain four equatorial pairs and two short axial pairs with atomic distances of about 2.0 and $1.4 \AA$ respectively, giving rise to an average distance of about $1.8 \AA$. For the tet- $\mathrm{O}$, the four $\mathrm{Fe}-\mathrm{O}$ pairs share equal distances of about $1.6 \AA$. Hence our experimental data, related precedent and energetic considerations all point to $\mathrm{O}$ inclusion within $b c c$-Fe on the octahedral interstitial site.

We developed a series of atomistic models for this $b c c-\mathrm{FeO}_{x}$ phase for compositions $0 \leq x \leq 1$. Here the oxygen stoichiometry $x$ reflects the charging capacity based on a nominal Li content " $\mathrm{Li}$ $-2 x$ ", i.e., a formula of " $\mathrm{FeO}_{0.5}$ " corresponds to the $\mathrm{Li}$ content of "Li -1.0 ". For selected $x$ values, we introduced local structure relaxation in our models by using an MMC algorithm to incorporate simple $\mathrm{O}-\mathrm{O}$ interactions. In the dilute solid solution $\left(\mathrm{FeO}_{0.1}\right)$, the MMC-derived oct-O distribution appears to be disordered (Supplementary Fig. 10). However, at the highconcentration limit $\left(\mathrm{FeO}_{1.0}\right)$, the driving force to minimise $\mathrm{O}-\mathrm{O}$ repulsion energies resulted in locally ordered domains (Fig. 4c). These domains all share the same short-range structure represented by a $b c c-\mathrm{FeO}$ unit cell (Fig. $4 \mathrm{~d}$ ) that contains a face and an edge $\mathrm{O}^{2-}$, isostructural to the tetragonal $\mathrm{FeO}(\mathrm{I} / \mathrm{mmm})^{34}$. The phase encrypts a superstructure that mirrors the tetragonally distorted $r s$ - $\mathrm{FeO}$ where the $\mathrm{Fe}$ sublattice retains the $b c c$ order, hinting at an underlying link between the $b c c$ - and $r s-\mathrm{FeO}$.

An important test of the physical sense of these models comes from their ability to account for the experimental PDF data. Consequently, our model PDFs were calculated using additional physical parameters, e.g., scale factor and linear correlation factor (Supplementary Fig. 11a), which were predefined via initial refinement against the Fe structure. The resulting PDFs (Fig. 4e) indeed exhibit the $\mathrm{Fe}-\mathrm{O}$ peak at around $2.0 \AA$ with growing intensity as oxygen content increases. Nearly all other variations observed experimentally as a function of composition are also well represented by our calculated PDFs. The overall agreement between simulation and experiment appears surprisingly good.
$\mathrm{Mn}_{3} \mathrm{O}_{4}$ (model compound for $\mathrm{Mn}_{\boldsymbol{x}} \mathrm{O}_{y}$ ). In contrast to $\alpha-\mathrm{Fe}_{2} \mathrm{O}_{3}$, the scattering patterns of $\mathrm{Mn}_{3} \mathrm{O}_{4}$ reflect the occurrence of a phase transition accompanied by significant $\mathrm{Mn}$ atomic rearrangement during charge. The broad width of the Bragg peaks (Supplementary Fig. 7) and the short coherence lengths $(<20 \AA)$ of the PDF patterns (Fig. 5a) indicate that the average grain sizes of Mncontaining species remain small during delithiation. Despite a two-step electrochemical process, which suggests the involvement of an intermediate, preliminary PDF refinements were attempted using a two-phase model consisting of $\alpha$-Mn $(I \overline{4} 3 \mathrm{~m})$ and $r s$ $\mathrm{MnO}(F m \overline{3} m)$. As anticipated, such refinements were unable to account satisfactorily for the data, demonstrating instead the necessity of including a third phase in the model. Given the analytic uncertainties concerning the intermediate, we implemented our recently developed method ${ }^{18}$ based on $\mathrm{NMF}^{35}$, a robust computational approach to study complex mixtures without a priori knowledge of the number and nature of each component. In the analysis, we employed three members each representing the starting, intermediate and end phases. The component representing the starting state was defined by the experimental pattern at the beginning of charge ("Li 0.0 "), while the other two were both set as variable components to be determined by the NMF analysis. The resulting PDFs of the two unknown members (Supplementary Movie 1) both show wellresolved patterns (Fig. 5b) with their respective weightings (Fig. 5c) following systematic evolutions that resonate with the two-step mechanism. The reconstructed PDF using the NMFderived output delivers a striking agreement with the experimental data $\left(R_{\mathrm{w}}<1.6 \%\right)$, confirming the credibility of the approach (Supplementary Fig. 12).

PDF refinement was then performed to rationalise the two newly extracted components. For the reconverted phase at the end of charge, its local ordering can be well modelled using a $(z b)$ $\mathrm{MnO}(F \overline{4} 3 m)^{36}$, whose (111) reflection contributes to the very intense Bragg peak at $2.3 \AA^{-1}$ in the XRD pattern (Fig. 2). Modern computational studies ${ }^{36-39}$ on the polymorphic landscape of $\mathrm{MnO}$ highlight the relative stability of the $z b$ phase $^{36-38}$ compared to the $r s$ ground-state structure; we also note the 
a

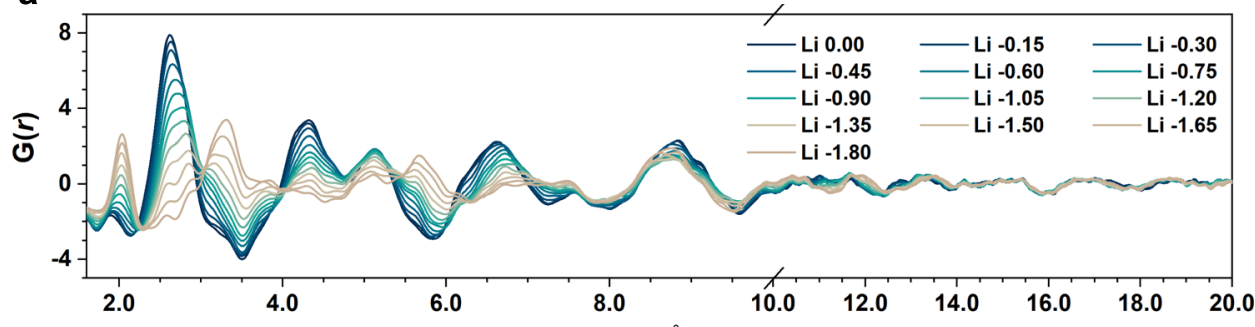

b

$r / \AA$

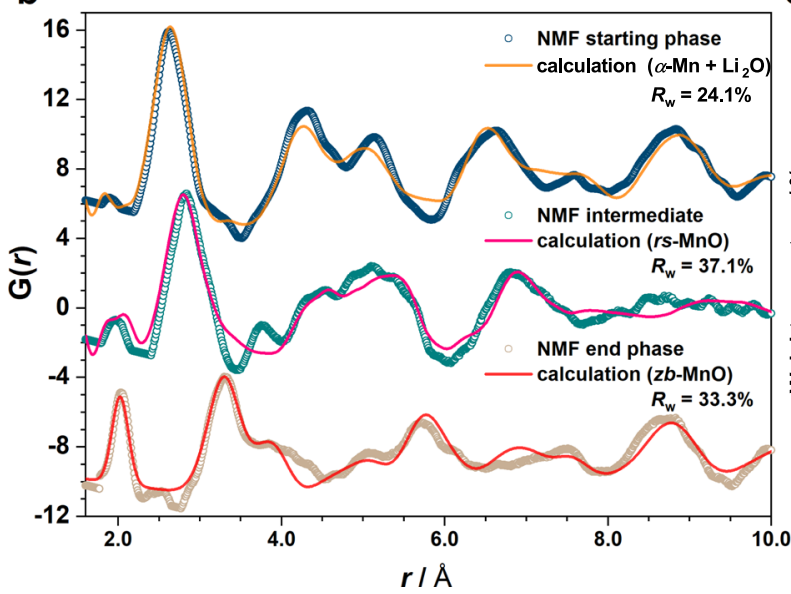

C 100

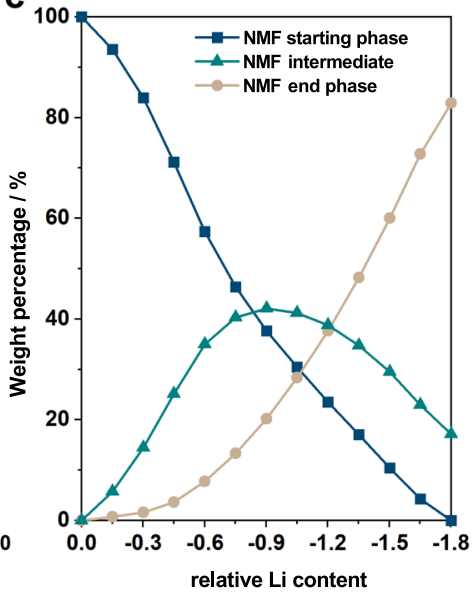

Fig. 5 NMF analysis of the charge process of $\mathbf{M n}_{\mathbf{3}} \mathbf{O}_{\mathbf{4}}$. a In situ PDF patterns for $\mathrm{Mn}_{3} \mathrm{O}_{4}$ during the first charge (plotted in two different $x$-scales for a clearer view). Colour gradient from dark blue ("Li $0.0^{\prime \prime}$ ) to light brown ("Li $-1.8^{\prime \prime}$ ) corresponds to a reducing concentration of Li. b Three NMF-derived

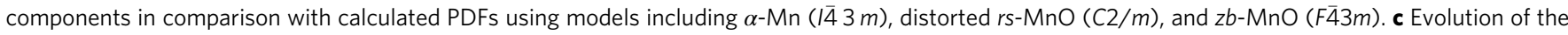
NMF-derived weight percentage for the components shown in (b).

experimental discovery of a related wurtzitic polymorph ${ }^{40}$. Our refined lattice constant of the $z b-\mathrm{MnO}, 4.687( \pm 0.006) \AA$, agrees excellently with the ab initio value $4.73( \pm 0.004) \AA^{38}$. For the intermediate component, its PDF fingerprint insinuates the Mncontaining atom pairs in an rs-related $\mathrm{MnO}$ (Supplementary Fig. 13), a phase that could also account for the scattering feature observed in the reciprocal space (Supplementary Fig. 7c). However, peak positions in real-space show discernible discrepancies implying distortion of the $\mathrm{MnO}_{6}$-octahedra ${ }^{7}$. To model this distortion, a monoclinically distorted $r s-\mathrm{MnO}(\mathrm{C} 2 / \mathrm{m})$ was employed in the refinement, noticeably improving the fit to the NMF-derived intermediate component. Note that as a phase experiencing a transition from $\alpha-\mathrm{Mn} / \mathrm{Li}_{2} \mathrm{O}$ to $z b-\mathrm{MnO}$, this intermediate is likely to remain lithiated. However, given the challenge in compositional determination, also considering the lack of structure periodicity and the likelihood of a heterogeneous cation distribution, it is difficult to reach a unique solution to fully model this intermediate. While our study concluded monoclinically distorted $r s-\mathrm{MnO}$ provides a good fit to its average shortrange ordering, we cannot rule out other possible, related models.

\section{Discussion}

Each of the phases $a f-\mathrm{Li}_{2} \mathrm{O}, r s$ - and $z b-\mathrm{MnO}$ adopt an $f c c-\mathrm{O}$ lattice. As the $\mathrm{Mn}_{x} \mathrm{O}_{y}$ PDFs show little variation in the coherent length of the system-indicating comparable domain sizes of the hosting structure throughout the charge and subsequent discharge steps-we can infer the oxygen ordering likely remains essentially intact despite significant changes in the cation distribution. On the basis of this preserved oxygen framework, Mn intercalation accompanied by concurrent $\mathrm{Li}$ extraction (or vice versa) appears to follow a topotactic insertion-like transformation, mechanistically referred to as a displacement reaction ${ }^{41}$.
Likewise, in the case of $\mathrm{Fe}_{x} \mathrm{O}_{y}$, the preservation of the $b c c$-Fe structure allows for its homogenous reaction with oxygen, implying the subsequent (dis)charge reactions of the Fe series are also diffusion-controlled, mechanistically similar to displacement, however, with $\mathrm{O}^{2-}$ now the displaced species. In both systems, this mechanistic transition exerts a tremendous influence on the reaction thermodynamics and kinetics of the subsequent cycles.

For $\mathrm{Fe}$ oxides, upon initial $\mathrm{Li}^{+}$withdrawal from $\mathrm{Li}_{2} \mathrm{O}$ (process I), the dissociated $\mathrm{O}^{2-}$ migrates to the $\mathrm{Li}_{2} \mathrm{O} / \mathrm{Fe}$ interface and diffuses within the Fe lattice (Fig. 6a). The inserted $\mathrm{O}^{2-}$ occupies the $b c c$-octahedral site, dispersing throughout the phase to reduce anion-anion repulsion. The potential presence of the Fe vacancy defects could further increase the affinity for the oct-O in the bcc$\mathrm{Fe}^{33}$. As the number of inserted $\mathrm{O}^{2-}$ increases, the lattice expands continuously-hence a gradual shift of the Fe Bragg peaks to a lower scattering angle (Supplementary Fig. 6). In contrast, the $\mathrm{Li}_{2} \mathrm{O}$ reflections shift in the opposite sense due to a lattice contraction as a result of either a declining number of interstitial $\mathrm{Li}^{+}$ and/or reduced grain size ${ }^{42}$. When the oxygen content $x$ reaches 0.5 , the driving force to minimise the $\mathrm{O}-\mathrm{O}$ interaction, hence the energy of $\mathrm{FeO}_{x}$ (Supplementary Fig. 14), promotes a disorder-order transition (process $I I)$ during which oct- $\mathrm{O}^{2-}$ rearranges to form an ordered sublattice. Upon further charging $(x>0.5)$, the structure becomes increasingly compact, which leads to progressively hindered $\mathrm{O}^{2-}$ diffusion as is consistent with the significantly increased overpotentials (Supplementary Fig. 15); ${ }^{43}$ this compact structure, on the other hand, results in an increasingly strained lattice. Without evident fracture of the $\mathrm{FeO}_{x}$ nanoparticles, this strain instead manifests itself as local Fe displacements, which account for the increasingly broadened peak widths and reduced intensities observed in the PDF (Supplementary Fig. 11a). During subsequent discharge, physical parameters derived from the refinement (Supplementary Fig. 11b) 

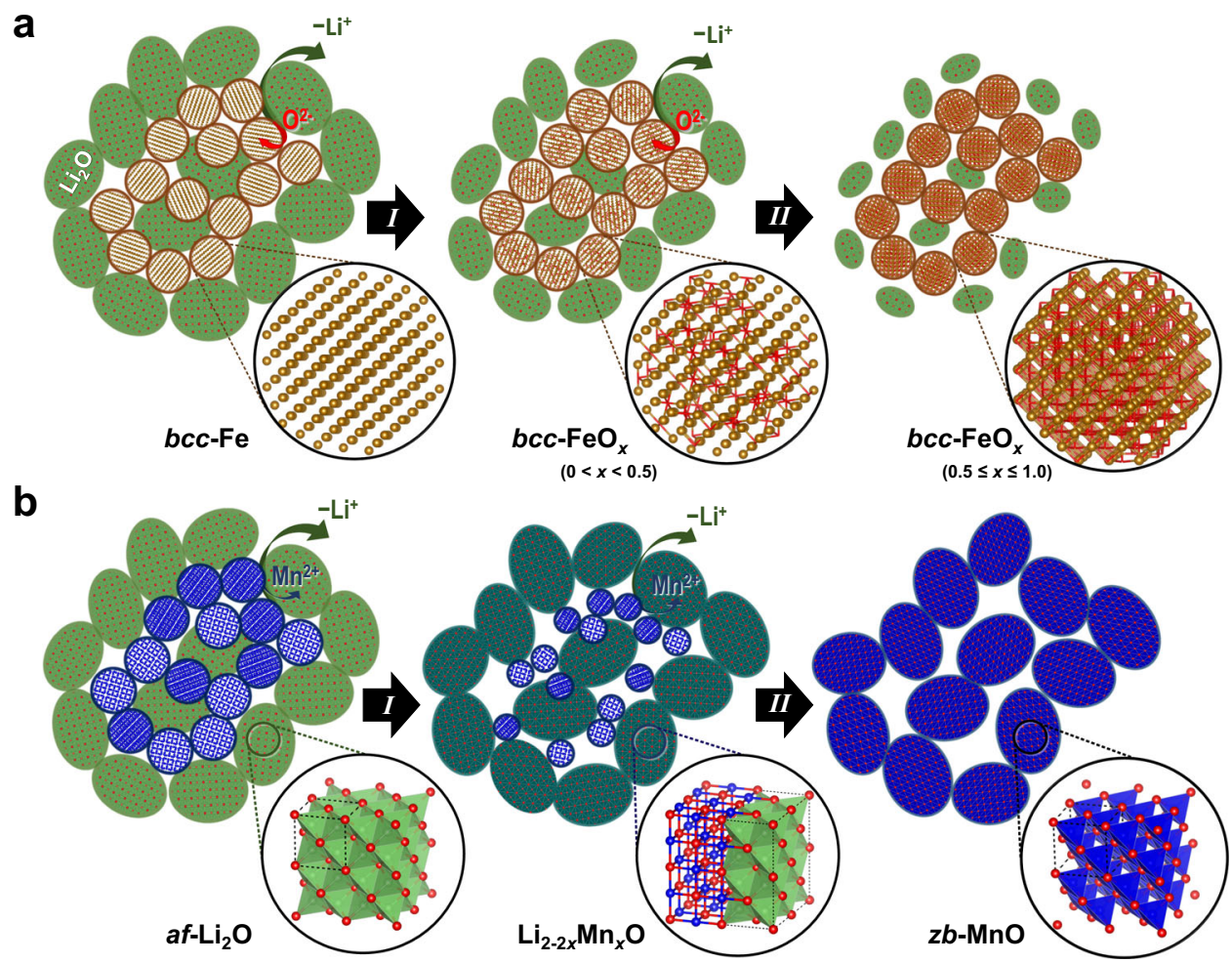

$(0<x<0.5)$

$(0.5 \leq x \leq 1.0)$
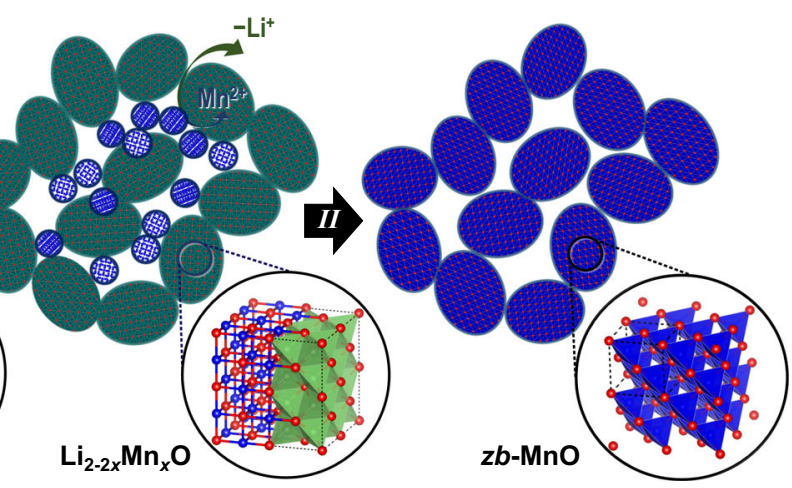

Fig. 6 Reaction mechanism of the charge process. Illustrated mechanisms of $\mathbf{a} \mathrm{Fe}_{x} \mathrm{O}_{y}$ and $\mathbf{b} \mathrm{Mn}_{x} \mathrm{O}_{y}$. Host structures are highlighted and magnified in black circles. $\mathrm{Fe}, \mathrm{Mn}, \mathrm{O}$, and $\mathrm{Li}$ are denoted by brown, blue, red, and green atoms/polyhedra. Yellow, blue, and green spheroids represent $\mathrm{Fe}$, $\mathrm{Mn}$, and $\mathrm{Li} \mathrm{i}_{2} \mathrm{O}$ particles, respectively. Cyan and blue ellipsoids in (b) represent $\mathrm{Li}_{2-2 x} \mathrm{Mn}_{x} \mathrm{O}$ and $\mathrm{MnO}$ particles, respectively. Note that $\mathrm{Li}$ and $\mathrm{Mn}$ ordering in the $\mathrm{Li}_{2-2 x} \mathrm{Mn}_{x} \mathrm{O}$ structure are shown in divided sections for a clearer view-it does not represent the actual cation distribution.

exhibit a progression that echoes the evolution of the parameters obtained from the charge step. This implies a reversible two-step process occurring during the second discharge, and hence a symmetrical thermodynamic pathway between the reversible cycles.

In contrast to $\mathrm{O}^{2-}$ insertion into the Fe-lattice, delithiation of $\mathrm{Mn}$ oxides takes place via the opposite route. This difference may arise in part due to the weaker metallic bond of $M n\left(3 d^{5} 4 s^{2}\right)$ that arises as a result of its half-filled $d$-orbitals. Upon charging, initial $\mathrm{Li}^{+}$extraction partly vacates the tet- $\mathrm{Li}$ sites in $a f-\mathrm{Li}_{2} \mathrm{O}$ while the oxidised $\mathrm{Mn}^{2+}$ dissociates from the metal nanoparticle and inserts into the octahedral vacancies in $\mathrm{Li}_{2} \mathrm{O}$ (Fig. 6b). This reaction (process $I$ ) results in an intermediate $\mathrm{Li}^{\text {tet }}{ }_{2-2 x} \mathrm{Mn}^{\text {oct }}{ }_{x} \mathrm{O}$ phase whose cations may adopt an anisotropic distribution, rendering its average local structure similar to a distorted $r s$-MnO. Surprisingly, upon further removal of $\mathrm{Li}^{+}, \mathrm{Mn}^{2+}$ does not remain within the octahedral sites to form the naturally occurring $r s-\mathrm{MnO}$; instead, it migrates to the tetrahedral positions (process $I I$ ) where the cation ordering is as in $z b$-ZnO. During subsequent discharge cycles, based on our NMF analysis (Supplementary Figs. 16 and 17), Mn nanoparticles appear to extrude directly from the $z b-\mathrm{MnO}$ (and the remaining intermediate) without going through any traceable transition step, rendering the discharge pathway asymmetric to the charging process. For both $\mathrm{Mn}$ and $\mathrm{Fe}$ systems, based on the reaction reversibility, they should in principle exhibit identical or comparable (given the asymmetric reaction path for $\mathrm{Mn}_{x} \mathrm{O}_{y}$ ) equilibrium voltages. Thus, very similar open-circuit voltages (OCVs) between the first charge and the second discharge steps would be expected in the galvanostatic intermittent titration (GITT) plot. However, a pronounced OCV gap within the reversible cycles is evident in the experimental data (Fig. 7, marked by green arrows), reflecting a deviation from the theoretical equilibrium. Such a deviation has

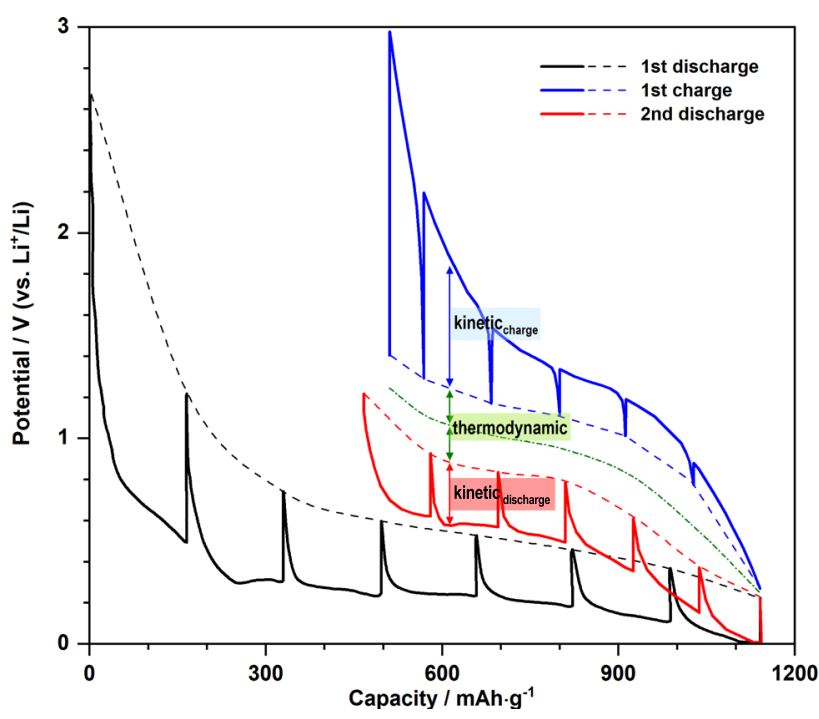

Fig. 7 Origin of the hysteresis in $\mathbf{M}_{\boldsymbol{x}} \mathbf{O}_{\boldsymbol{y}}$. Galvanostatic intermittent titration (GITT) result from $\mathrm{MnO}$ measured by using a $\mathrm{C} / 20$ current rate and 64-h relaxation time (adapted from literature ${ }^{21}$ ). Solid and dash curves, respectively, denote the operating voltages and the OCVs during the first discharge (black), first charge (blue), and second discharge (red) steps. Green dash-dot curve marks the average of the OCVs between the $1^{\text {st }}$ charge and $2^{\text {nd }}$ discharge, representing the theoretical equilibrium voltage during the reversible cycles. The overpotentials upon the first charge and second discharge, which contribute to the kinetic hysteresis, are labelled by blue and red arrows, respectively. The voltage difference between the OCV (red/blue dash) and the equilibrium voltage (green dash-dot), which constitute the thermodynamic hysteresis, is labelled by green arrows. 
a thermodynamic origin and could be largely due to the voltage modification as a result of inherent "zero-current" hystersis ${ }^{44}$. In addition, an earlier study observed that an increase in surface tension due to the particle size reduction could increase the OCV gap $^{45}$. Given the inevitable electrochemical grinding of $M_{x} \mathrm{O}_{y}$ into ever smaller particles, their large surface energy might be another intrinsic factor contributing to this thermodynamic hysteresis.

Concerning kinetics, a displacement-like mechanism imparts a substantial dependence on the relative transport properties of different displaced species. As the discharge and charge overpotentials in both systems show an asymmetric response (Fig. 7 and Supplementary Fig. 15) - constituting a path hysteresis ${ }^{46}$ typical for displacement mechanism-we expect the mobilities of $\mathrm{Mn}$ and $\mathrm{O}$ in their respective host structures to be generally lower than $\mathrm{Li}$ diffusion in most cases. Hence, the discharge kinetics during the reversible cycles for both $\mathrm{Mn}$ and Fe systems is likely to be limited by $\mathrm{Mn} / \mathrm{O}$ extraction from their hosts leading to a relatively small degree of variation in the discharge polarisation. By contrast, upon charge, the mobility difference between Li and $\mathrm{Mn} / \mathrm{O}$ would result in more rapid $\mathrm{Li}$ removal than $\mathrm{Mn} / \mathrm{O}$ insertion, which we observe as gradually increased overpotentials as lithium is removed. It is likely this asymmetric path hysteresis-in conjunction with other effects, such as passivation layers ${ }^{11}$ (hence sluggish interface mobilities) - plays a key role in modifying the voltage polarisation. The build-up of the hysteresis from both thermodynamic and kinetic origins eventually manifests itself as a voltage gap that looks unexpectedly large compared to that of conventional insertion materials. Having said that, $M_{x} \mathrm{O}_{y}$ 's hysteretic behaviour during the reversible cycles is notably reduced relative to that of the first cycle. It is also worth noting that the asymmetric properties in both thermodynamic (i.e., two-step charge vs one-step discharge) and kinetic pathways of the Mn system jointly give rise to its asymmetric (operating) voltage profile in which the discharge curve generally shows a prominent (pseudo-)plateau. By contrast, this effect is less pronounced in the Fe systems where both discharge and charge curves during reversible cycles appear more slope-like (Supplementary Fig. 1b).

Our results offer insights to guide the future improvement of metal oxides. Firstly, they imply that the key factors that influence the voltage polarisation are primarily mechanism-dependentthus intrinsic to the materials. Hence, nanostructure engineering may have relatively little impact on the mitigation of the observed hysteretic behaviour ${ }^{43}$. That being said, $M_{x} \mathrm{O}_{y}$ with unique structure designs ${ }^{47}$ - particularly when mechanically reinforced by carbon 20,48 -might have macro- and microscopic physical features such as structural hierarchy and mesoporosity that nonetheless survive the destructive pulverisation of the first discharge. The preserved structure integrity remains important in the subsequent cycling as it could ensure both effective spatial distribution between $M$ and $\mathrm{Li}_{2} \mathrm{O}$ as well as adequate electrolyte permeability for Li reaction. As a result, structure-engineered 47,48 $M_{x} \mathrm{O}_{y}$ is more likely to surpass non-structured ${ }^{11}$ materials (i.e., commercial materials) in rate performance and capacity retention (Supplementary Fig. 1b). To this end, effective morphology design remains important to harness the full potential of $M_{x} \mathrm{O}_{y}$ phases. Secondly, the $2 e^{-}$transfer pathway (per $M$ centre) during reversible cycling and thus the obtainable capacity (i.e., excluding the contribution from side reactions) within the regular voltage window $\left(0-3 \mathrm{~V}\right.$ vs $\left.\mathrm{Li}^{+} / \mathrm{Li}\right)$ appear insensitive to the $M_{x} \mathrm{O}_{y}$ composition. Therefore, although $M_{x} \mathrm{O}_{y}$ phases with high $M$ oxidation states deliver a larger capacity in the first discharge, they also produce an excess of $\mathrm{Li}_{2} \mathrm{O}$, which will remain inactive during subsequent cycles. Not only is this additional capacity inaccessible thereafter, but the excess $\mathrm{Li}_{2} \mathrm{O}$ also exacerbates volume expansion during the first discharge-in turn impairing the mechanical integrity of the electrode ${ }^{49}$. Moreover, the electronically insulating effect of $\mathrm{Li}_{2} \mathrm{O}$ and its accumulation during the following cycles may lead to further capacity fading ${ }^{11}$. Hence, to effectively minimise the inherent formation of excess $\mathrm{Li}_{2} \mathrm{O}$, future material development should involve strategic selection of $M_{x} \mathrm{O}_{y}$ phases with lower metal oxidation states: i.e., $\mathrm{FeO} / \mathrm{MnO}>\mathrm{Fe}_{3} \mathrm{O}_{4} /$ $\mathrm{Mn}_{3} \mathrm{O}_{4}>\mathrm{Fe}_{2} \mathrm{O}_{3} / \mathrm{Mn}_{2} \mathrm{O}_{3}>\mathrm{MnO}_{2}$. An alternative strategy to better control the $M: \mathrm{Li}_{2} \mathrm{O}$ ratio is to fabricate $M / \mathrm{Li}_{2} \mathrm{O}$ nano-mixtures as the pre-lithiated active material. This proof of concept has been proven viable in earlier studies where non-engineered $M / \mathrm{Li}_{2} \mathrm{O}$ nanocomposites were used as cathode additives ${ }^{50}$. It is worth exploring the application to anodes, with future effort directed towards carbon mixing and effective morphology engineering. Thirdly, from a broader perspective, the general interest in conversion metal oxides extends from binary to ternary phases ${ }^{51}$ and includes other 1st-row transition metals and beyond, e.g., $\mathrm{Ni}(f c c)$, Co (hcp), and $\mathrm{Zn}(h c p)$; we also noticed a change of $\mathrm{Mn}_{x} \mathrm{O}_{y}$ cycling behaviour in the literature due to an unintentional mixing with the $\mathrm{Ni}$ template ${ }^{52}$. In light of the mechanistic differences between the $\mathrm{Mn}$ and Fe systems observed here, we envisage that other binary metal oxides may exhibit quite unexpected electrochemical properties-given the distinct crystallographic and physicochemical properties of each metal. It will, therefore, be important to revisit these $M_{x} \mathrm{O}_{y}$ systems (possibly with supplementary XAS techniques to enhance the knowledge of their electronic structures), such that an improved and systematic understanding of (i) their generic reversible phase behaviours upon cycling and (ii) diffusion properties of different displaced species is gained. This will uncover synergies from different $M_{x} \mathrm{O}_{y}$ and hence enable the design of future materials with complex compositions that are doped with displaced species with fast mobilities, e.g., ternary systems.

As a final point we note that, while our focus has been on elucidating $M_{x} \mathrm{O}_{y}$ 's electrochemical transformation pathways, we do also report the first experimental observation of the nonequilibrium phases $b c c-\mathrm{FeO}$ and $z b-\mathrm{MnO}$. In their ground states, both $\mathrm{FeO}$ and $\mathrm{MnO}$ are known to adopt the rs structure. Accessing other polymorphs normally requires high-temperature and/or high-pressure routes ${ }^{53,54}$. A recent attempt using hydrothermal methods successfully achieved nanostructured wurtzitic $\mathrm{MnO}^{40}$, demonstrating the viability to synthesise non-native $\mathrm{MO}$ polymorphs without extreme conditions. As a result of electrochemical pulverisation, the $M / \mathrm{Li}_{2} \mathrm{O}$ nanocomposites left after discharge possess a large surface energy. Under well-defined electrochemical conditions, this substantial surface energy can stabilise structure types that are otherwise kinetically or thermodynamically unstable, and hence allow the formation of new $M O$ polymorphs. As $M O$ with different structures exhibits interesting physicochemical properties, concerning the significance of $M O$ in a wide range of fields, including but not limited to chemical engineering ${ }^{55}$, geoscience ${ }^{53,54}$, condensed matter physics ${ }^{36,56}$ and metallurgy ${ }^{33,57}$, the investigation of $M O$ and its polymorphism has become one of the most active areas in modern solid-state chemistry. In this way, electrochemical devices may offer an alternative synthesis strategy to explore non-native metal monoxides with new functionalities-e.g., the unique formation pathway of $b c c-\mathrm{FeO}$ is based on diffusion of $\mathrm{O}^{2-}$ in a close-packed Fe structure, indicating $\mathrm{Fe}$ nanoparticles could function as a metallic ionic conductor for $\mathrm{O}^{2-}$, a rare property for Fe.

To summarise, we have used a combination of in operando/ in situ PDF measurements, MMC simulations, and NMF analysis to elucidate the cycling mechanisms of $\mathrm{Fe}_{x} \mathrm{O}_{y}$ and $\mathrm{Mn}_{x} \mathrm{O}_{y}$ phases following first discharge. The mechanism adopted depends on the choice of transition metal but is insensitive to the composition of the starting $M_{x} \mathrm{O}_{y}$. Although a one-step conversion reaction from $M$ to $r s$-MO has been widely accepted as the delithiation pathway 
upon charge given their similar voltage profiles, our study has shown that both $\mathrm{Fe}$ and $\mathrm{Mn}$ oxides exhibit a two-step mechanism via displacement-like reactions forming non-equilibrium $b c c-\mathrm{FeO}$ and $z b-\mathrm{MnO}$ phases. Importantly, while this topotactic behaviour in $\mathrm{Mn}_{x} \mathrm{O}_{y}$ manifests as intercalation of $\mathrm{Mn}^{2+}$ into the $f c c-\mathrm{O}$ sublattice, a different pathway based on $\mathrm{O}^{2-}$ insertion into the $b c c$-Fe sublattice was observed in the Fe system. For both materials-based on their distorted voltage polarisations within the reversible cycles-we show that the path hysteresis originates from the mobility differences amongst displaced species. Given the reversible cycles (within the regular voltage window) only involve $2 e^{-}$transfer (per metal centre), future material design should be directed towards systems that can demonstrate better control over excess $\mathrm{Li}_{2} \mathrm{O}$ produced during the first discharge. In light of the new MO polymorphs reported in this work, electrochemical syntheses may offer exciting opportunities to the discovery of new MO materials. Finally, this work demonstrates the viability of using our newly developed analytical approach combining with in situ PDF experiments to investigate battery materials with highly heterogeneous or amorphous structures.

\begin{abstract}
Methods
Materials. All $\mathrm{Fe}_{x} \mathrm{O}_{y}$ and $\mathrm{Mn}_{x} \mathrm{O}_{y}$ materials were purchased from Sigma-Aldrich. The electrode pellets that contain $70 \mathrm{wt} \% M_{x} \mathrm{O}_{y}$ active material, $10 \mathrm{wt} \%$ PTFE and 20 wt\% Super P carbon (TIMCAL C65) were prepared in the atmosphere. Swagelok-type in situ cells were assembled in Ar-filled glovebox (MBraun) using the as-prepared electrode pellets with a glass fibre (Whatman) as the separator, an $\mathrm{Li}$ metal foil as the counter electrode, and $1 \mathrm{M} \mathrm{LiPF}_{6}$ in ethylene carbonate (EC)/ dimethyl carbonate (DMC) solution (volume ratio 1:1) as the electrolyte. The fully discharged and charged $\alpha-\mathrm{Fe}_{2} \mathrm{O}_{3}$ materials for ex situ TEM measurement were cycled in coin cells. The coin cells were made in the same way as the in situ cells apart from the fact that $\alpha-\mathrm{Fe}_{2} \mathrm{O}_{3}$ powder was directly employed as electrode without additional carbon or PTFE. The cells were cycled at room temperature under a rate of $\mathrm{C} / 15$. They were stopped at 0 and $3 \mathrm{~V}$, respectively, for the discharged and charged samples. To prepare the TEM samples, these cells were transported and disassembled inside the Ar-filled glovebox. After disassembling, the materials were rinsed with DMC twice before drying in the antechamber.
\end{abstract}

Characterisation. The in situ X-ray total scattering data were collected at beamline I15-1 at the Diamond Light Source using an amorphous silicon area detector (Perkin Elmer, XRD4343CT) with an X-ray beam of energy $76.69 \mathrm{keV}(\lambda=0.1617$ $\AA$ ) and $Q_{\max }$ of $25 \AA^{-1}$. Experiments were conducted in transmission geometry within perfluoroalkoxy alkane (PFA) Swagelok cells. Empty Swagelok cells were measured for the background of every in situ cell. The in situ electrochemistry was conducted using a rate of $\mathrm{C} / 15$ for every cell. Data reduction and normalisation were performed using DAWN ${ }^{58}$ and PDFgetX2 ${ }^{59}$, respectively. An additional $\mathrm{LaB}_{6}$ pattern was collected as a reference to obtain instrumental damping factor for PDF refinement. The PDF refinements were performed using the PDFgui ${ }^{60}$ software. For TEM measurements immediately following preparation of the ex situ samples, we used a JEOL $3000 \mathrm{~F}$ Field emission gun TEM with an air-tight TEM holder.

MMC simulation. MMC simulations ${ }^{17}$ were performed on a supercell constructed using $7 \times 7 \times 7 \alpha$-Fe unit cell with the periodic boundary conditions applied. The dimensions of the box were determined based on the $2 \mathrm{~nm}$ size of the $\alpha$-Fe particles suggested by PDF. The number of oxygen atoms was determined by the Fe:O ratio in $\mathrm{FeO}_{x}$ and were first randomly distributed into the oct-sites of the $b c c$-Fe. The $\mathrm{MMC}$ energy was calculated on the basis of repulsion between oxygen considering the O-O interaction within the three nearest coordination shells of each oxygen. At each MMC step, a randomly selected oxygen atom was swapped with another randomly selected empty oct-site. The energy change $(\Delta E)$ due to the move was calculated. The move was automatically accepted if $\Delta E \leq 0$; for a positive energy change, the acceptance was subject to the Metropolis algorithm ${ }^{17}$ given by $P=e^{-\Delta E / k T}$, where $k$ is the Boltzmann constant and $T$ is the temperature. Moves were continuously proposed, and accepted or rejected until convergence was achieved.

Non-negative matrix factorisation. The NMF approach followed closely the Metropolis Matrix Factorisation (MMF) method reported earlier ${ }^{18}$, which uses the MMC algorithm to carry out $\mathrm{NMF}^{35}$. The NMF analysis was performed on renormalised PDFs to satisfy the non-negative criterion of NMF. The renormalised $g^{\exp }(r)$ were derived from the experimental $G^{\exp }(r)$ using the equation $G(r)=4 \pi r \rho_{0}(g(r)-1)^{61}$, in which $\rho_{0}$ refers to the number density of the structure model. Three fundamental components $g_{i}^{*}(r)(i=3)$ were employed in the analysis. The goal of the analysis was to identify these $g_{i}^{*}(r)$ and associated weights $w_{i j}$ (j corresponds to the number of experimental $g_{j}^{\exp }(r)$ ) to minimise $\left|g_{j}^{\text {calc }}(r)-g_{j}^{\exp }(r)\right|^{2}$, where $g_{j}^{\text {calc }}(r)=\sum_{i=1}^{3} w_{\mathrm{ij}} g_{i}^{*}(r)$. Additional constraints were applied to ensure non-negative $g_{i}^{*}(r)$ for all $i$ and $r$, and that $\sum_{i=1}^{3} w_{\mathrm{ij}}=1$ for all $j$. The initial $g_{1}^{*}(r)$ representing the known component was fixed as the experimental function measured at the end of first discharge, whereas the two unknown components $g_{2}^{*}(r)$ and $g_{3}^{*}(r)$ and all $w_{i j}$ were assigned randomly subject to the various constraints listed above. Each iteration involved a random variation of these parameters, followed by the calculation of the change in $\left|g_{j}^{\text {calc }}(r)-g_{j}^{\exp }(r)\right|^{2}$. The acceptance or rejection of the variation follows MMC algorithm. The variation was repeated under increasingly stringent acceptance criteria using simulated annealing until convergence was achieved.

\section{Data availability}

The authors declare that all data supporting the findings of this study are included within the paper and its Supplementary Information files. Source data are available from the corresponding author upon reasonable request.

Received: 20 August 2020; Accepted: 7 December 2020; Published online: 25 January 2021

\section{References}

1. Cabana, J., Monconduit, L., Larcher, D. \& Palacín, M. R. Beyond intercalationbased Li-ion batteries: the state of the art and challenges of electrode materials reacting through conversion reactions. Adv. Mater. 22, E170-E192 (2010).

2. Yu, S.-H., Lee, S. H., Lee, D. J., Sung, Y.-E. \& Hyeon, T. Conversion reactionbased oxide nanomaterials for lithium ion battery anodes. Small 12, 2146-2172 (2016)

3. Livage, J., Henry, M. \& Sanchez, C. Sol-gel chemistry of transition metal oxides. Prog. Solid State Chem. 18, 259-341 (1988).

4. Ren, Y., Ma, Z. \& Bruce, P. G. Ordered mesoporous metal oxides: synthesis and applications. Chem. Soc. Rev. 41, 4909-4927 (2012).

5. Butala, M. M. et al. MnO conversion in Li-ion batteries: in situ studies and the role of mesostructuring. ACS Appl. Mater. Interfaces 8, 6496-6503 (2016).

6. Yonekura, D. et al. Progress of the conversion reaction of $\mathrm{Mn}_{3} \mathrm{O}_{4}$ particles as a function of the depth of discharge. Phys. Chem. Chem. Phys. 16, 6027-6032 (2014).

7. Lowe, M. A., Gao, J. \& Abruna, H. D. In operando X-ray studies of the conversion reaction in $\mathrm{Mn}_{3} \mathrm{O}_{4}$ lithium battery anodes. J. Mater. Chem. A 1, 2094-2103 (2013).

8. Bock, D. C. et al. Size dependent behavior of $\mathrm{Fe}_{3} \mathrm{O}_{4}$ crystals during electrochemical (de)lithiation: an in situ X-ray diffraction, ex situ X-ray absorption spectroscopy, transmission electron microscopy and theoretical investigation. Phys. Chem. Chem. Phys. 19, 20867-20880 (2017).

9. Su, Q., Xie, D., Zhang, J., Du, G. \& Xu, B. In situ transmission electron microscopy observation of the conversion mechanism of $\mathrm{Fe}_{2} \mathrm{O}_{3}$ /graphene anode during lithiation-delithiation processes. ACS Nano 7, 9115-9121 (2013).

10. Shyam, B. et al. Structural and mechanistic revelations on an iron conversion reaction from pair distribution function analysis. Angew. Chem. Int. Ed. 51, 4852-4855 (2012).

11. Li, J. et al. Phase evolution of conversion-type electrode for lithium ion batteries. Nat. Commun. 10, 2224 (2019).

12. Zhang, W. et al. Insights into ionic transport and structural changes in magnetite during multiple-electron transfer reactions. Adv. Energy Mater. 6, 1502471 (2016).

13. Penner-Hahn, J. E. in eLS (John Wiley \& Sons, Ltd, 2001).

14. Britto, S. et al. Multiple redox modes in the reversible lithiation of highcapacity, peierls-distorted vanadium sulfide. J. Am. Chem. Soc. 137, 8499-8508 (2015).

15. Hu, Y.-Y. et al. Origin of additional capacities in metal oxide lithium-ion battery electrodes. Nat. Mater. 12, 1130-1136 (2013).

16. Hua, $\mathrm{X}$. et al. Comprehensive study of the $\mathrm{CuF}_{2}$ conversion reaction mechanism in a lithium ion battery. J. Phys. Chem. C 118, 15169-15184 (2014).

17. Metropolis, N., Rosenbluth, A. W., Rosenbluth, M. N., Teller, A. H. \& Teller, E. Equation of state calculations by fast computing machines. J. Chem. Phys. 21, 1087-1092 (1953).

18. Geddes, H. S., Blade, H., McCabe, J. F., Hughes, L. P. \& Goodwin, A. L. Structural characterisation of amorphous solid dispersions via metropolis matrix factorisation of pair distribution function data. Chem. Commun. 55, 13346-13349 (2019). 
19. Gao, M. et al. FeO/C anode materials of high capacity and cycle stability for lithium-ion batteries synthesized by carbothermal reduction. J. Alloy. Compd. 565, 97-103 (2013).

20. Jiang, Y., Jiang, Z.-J., Yang, L., Cheng, S. \& Liu, M. A high-performance anode for lithium ion batteries: $\mathrm{Fe}_{3} \mathrm{O}_{4}$ microspheres encapsulated in hollow graphene shells. J. Mater. Chem. A 3, 11847-11856 (2015).

21. Zhong, $\mathrm{K}$. et al. $\mathrm{MnO}$ powder as anode active materials for lithium ion batteries. J. Power Sources 195, 3300-3308 (2010).

22. Gao, J., Lowe, M. A. \& Abruña, H. D. Spongelike nanosized $\mathrm{Mn}_{3} \mathrm{O}_{4}$ as a highcapacity anode material for rechargeable lithium batteries. Chem. Mater. 23, 3223-3227 (2011).

23. Xia, H., Lai, M. \& Lu, L. Nanoflaky $\mathrm{MnO}_{2} /$ carbon nanotube nanocomposites as anode materials for lithium-ion batteries. J. Mater. Chem. 20, 6896-6902 (2010).

24. West, A. R. \& Bruce, P. G. Tetragonal-packed crystal structures. Acta Crystallogr. B 38, 1891-1896 (1982).

25. $\mathrm{Ma}, \mathrm{X}$. et al. Inheritance of crystallographic orientation during lithiation/ delithiation processes of single-crystal $\alpha-\mathrm{Fe}_{2} \mathrm{O}_{3}$ nanocubes in lithium-ion batteries. ACS Appl. Mater. Interfaces 7, 24191-24196 (2015).

26. Qin, F. et al. Sustainable synthetic route for $\gamma-\mathrm{Fe}_{2} \mathrm{O}_{3} / \mathrm{C}$ hybrid as anode material for lithium-ion batteries. Dalton Trans. 44, 2150-2156 (2015).

27. Deng, $\mathrm{Y}$. et al. Porous $\mathrm{Mn}_{2} \mathrm{O}_{3}$ microsphere as a superior anode material for lithium ion batteries. RSC Adv. 2, 4645-4647 (2012).

28. Tang, X., Sui, G., Cai, Q., Zhong, W. \& Yang, X. Novel MnO/carbon composite anode material with multi-modal pore structure for high performance lithium-ion batteries. J. Mater. Chem. A 4, 2082-2088 (2016).

29. Kim, B., Sietsma, J. \& Santofimia, M. J. Thermodynamic aspects of carbon redistribution during ageing and tempering of Fe-Ni-C alloys. Philos. Mag 96, 2632-2648 (2016).

30. Van Genderen, M. J., Böttger, A. \& Mittemeijer, E. J. Formation of $\alpha^{\prime \prime}$ iron nitride in FeN martensite: nitrogen vacancies, iron-atom displacements, and misfit-strain energy. Metall. Mater. Trans. 28, 63-77 (1997).

31. Souissi, M. \& Numakura, H. Elastic properties of Fe-C and Fe-N martensites. ISIJ Int. 55, 1512-1521 (2015).

32. Jiang, D. E. \& Carter, E. A. Diffusion of interstitial hydrogen into and through bcc Fe from first principles. Phys. Rev. B 70, 064102 (2004).

33. Shang, S. L. et al. Vacancy mechanism of oxygen diffusivity in bcc Fe: a firstprinciples study. Corros. Sci. 83, 94-102 (2014).

34. Jain, A. et al. Commentary: the materials project: a materials genome approach to accelerating materials innovation. APL Mater. 1, 011002 (2013).

35. Lee, D. D. \& Seung, H. S. Learning the parts of objects by non-negative matrix factorization. Nature 401, 788-791 (1999).

36. Schrön, A., Rödl, C. \& Bechstedt, F. Energetic stability and magnetic properties of $\mathrm{MnO}$ in the rocksalt, wurtzite, and zinc-blende structures: influence of exchange and correlation. Phys. Rev. B 82, 165109 (2010).

37. Lanatà, N., Lee, T.-H., Yao, Y.-X., Stevanović, V. \& Dobrosavljević, V. Connection between Mott physics and crystal structure in a series of transition metal binary compounds. Npj Comput. Mater. 5, 30 (2019).

38. Schiller, J. A., Wagner, L. K. \& Ertekin, E. Phase stability and properties of manganese oxide polymorphs: assessment and insights from diffusion Monte Carlo. Phys. Rev. B 92, 235209 (2015).

39. Peng, H. \& Lany, S. Polymorphic energy ordering of $\mathrm{MgO}, \mathrm{ZnO}, \mathrm{GaN}$, and $\mathrm{MnO}$ within the random phase approximation. Phys. Rev. B 87, 174113 (2013).

40. Nam, K. M. et al. New crystal structure: synthesis and characterization of hexagonal wurtzite MnO. J. Am. Chem. Soc. 134, 8392-8395 (2012).

41. Goodenough, J. B. \& Park, K.-S. The Li-ion rechargeable battery: a perspective. J. Am. Chem. Soc. 135, 1167-1176 (2013).

42. Qi, W. H., Wang, M. P. \& Su, Y. C. Size effect on the lattice parameters of nanoparticles. J. Mater. Sci. Lett. 21, 877-878 (2002).

43. $\mathrm{Xu}, \mathrm{Y}$. et al. Superior electrochemical performance and structure evolution of mesoporous $\mathrm{Fe}_{2} \mathrm{O}_{3}$ anodes for lithium-ion batteries. Nano Energy 3, 26-35 (2014).

44. Dreyer, W. et al. The thermodynamic origin of hysteresis in insertion batteries. Nat. Mater. 9, 448-453 (2010).

45. Zhu, C. et al. Lithium potential variations for metastable materials: case study of nanocrystalline and amorphous $\mathrm{LiFePO}_{4}$. Nano Lett. 14, 5342-5349 (2014).

46. Yu, H. C. et al. Designing the next generation high capacity battery electrodes. Energy Environ. Sci. 7, 1760-1768 (2014).

47. $\mathrm{Xu}, \mathrm{S}$. et al. $\mathrm{\alpha}-\mathrm{Fe}_{2} \mathrm{O}_{3}$ multi-shelled hollow microspheres for lithium ion battery anodes with superior capacity and charge retention. Energy Environ. Sci. 7, 632-637 (2014).

48. Jiang, T. et al. Porous $\mathrm{Fe}_{2} \mathrm{O}_{3}$ nanoframeworks encapsulated within threedimensional graphene as high-performance flexible anode for lithium-ion battery. ACS Nano 11, 5140-5147 (2017).

49. Klein, F., Jache, B., Bhide, A. \& Adelhelm, P. Conversion reactions for sodiumion batteries. Phys. Chem. Chem. Phys. 15, 15876-15887 (2013).
50. Sun, Y. et al. High-capacity battery cathode prelithiation to offset initial lithium loss. Nat. Energy 1, 15008 (2016).

51. Bresser, D., Passerini, S. \& Scrosati, B. Leveraging valuable synergies by combining alloying and conversion for lithium-ion anodes. Energy Environ. Sci. 9, 3348-3367 (2016).

52. Liu, S. et al. Three dimensional hierarchically porous crystalline $\mathrm{MnO}_{2}$ structure design for a high rate performance lithium-ion battery anode. RSC Adv. 6, 85222-85229 (2016)

53. Ozawa, H., Takahashi, F., Hirose, K., Ohishi, Y. \& Hirao, N. Phase transition of FeO and stratification in earth's outer core. Science 334, 792 (2011).

54. Noguchi, Y., Kusaba, K., Fukuoka, K. \& Syono, Y. Shock-induced phase transition of $\mathrm{MnO}$ around 90GPa. Geophys. Res. Lett. 23, 1469-1472 (1996).

55. Heracleous, E. et al. Bio-oil upgrading via vapor-phase ketonization over nanostructured $\mathrm{FeO}_{\mathrm{x}}$ and $\mathrm{MnO}_{\mathrm{x}}$ : catalytic performance and mechanistic insight. Biomass. Convers. Biorefin. 7, 319-329 (2017).

56. Gillen, R. \& Robertson, J. Accurate screened exchange band structures for the transition metal monoxides $\mathrm{MnO}, \mathrm{FeO}, \mathrm{CoO}$ and $\mathrm{NiO}$. J. Phys. Condens. Matter 25, 165502 (2013).

57. Ostrovski, O., Olsen, S. E., Tangstad, M. \& Yastreboff, M. Kinetic modelling of $\mathrm{MnO}$ reduction from manganese ore. Can. Metall. Q. 41, 309-318 (2002).

58. Basham, M. et al. Data Analysis WorkbeNch (DAWN). J. Synchrotron Radiat. 22, 853-858 (2015).

59. Qiu, X., Thompson, J. W. \& Billinge, S. L. J. PDFgetX2: a GUI-driven program to obtain the pair distribution function from X-ray powder diffraction data. $J$. Appl. Crystallogr. 37, 678 (2004).

60. Farrow, C. L. et al. PDFfit2 and PDFgui: computer programs for studying nanostructure in crystals. J. Phys. Condens. Matter 19, 335219 (2007).

61. Egami, T. \& Billinge, S. J. L. Underneath the Bragg Peaks Structural Analysis of Complex Materials. Pergamon Materials Series 2nd edn, Vol. 16 (Elsevier, Pergamon, Kidlington, Oxford, 2012).

\section{Acknowledgements}

This work was supported by the European Commission via the MSCA-IF-2020. The use of Diamond Light Source for access to beamline I15-1 (XPDF) is under Proposal CY22115-1 and the use of TEM at the DCCEM, Department of Materials, Oxford, is supported by the Henry Royce Institute (Grant ref EP/R010145/1). X.H. acknowledges the funding from the European Commission via the MSCA (Grant 798169, DisorMetox). P.K.A. acknowledges a Birmingham Fellowship from the University of Birmingham. P.G.B. is indebted to the EPSRC for financial support including a Programme Grant, to the Faraday Institution and the Sir Henry Royce Institute. H.S.G. acknowledges the funding from the EPSRC via the CASE studentship and the Faraday Challenge projects FutureCat (Grant number FIRG017). A.L.G. acknowledges funding from the ERC (Grant 788144). The authors also acknowledge the helpful discussion and comments from $\mathrm{T}$. Dean, E. Reynolds, H. Liu, S. Britto, R. House, D. Forstermann, S. Booth, A. Minelli, E. Wolpert, E. Castillo-Martinez, S. Corr and S. Dutton.

\section{Author contributions}

X.H. conceived the study and designed the project. X.H. planned the project with the help from P.K.A. and A.L.G. X.H. prepared the materials for the project. X.H. acquired and analysed the electrochemical data with the help from P.K.A. and the support from P. G.B. X.H. and P.K.A. carried out the in situ PDF experiments with the help from P.A.C. C.G. acquired and analysed the TEM data under the supervision from A.W.R. X.H. performed structure modelling with the help from E.M.S. and A.L.G. X.H. performed the NMF analysis with the help from H.S.G. and A.L.G. The manuscript was written by X.H and revised by P.K.A. and A.L.G. All authors contributed to discussions and commented on the manuscript.

\section{Competing interests}

The authors declare no competing interests.

\section{Additional information}

Supplementary information is available for this paper at https://doi.org/10.1038/s41467020-20736-6.

Correspondence and requests for materials should be addressed to X.H.

Peer review information Nature Communications thanks Anatoly Frenkel and the other, anonymous, reviewer(s) for their contribution to the peer review of this work.

Reprints and permission information is available at http://www.nature.com/reprints

Publisher's note Springer Nature remains neutral with regard to jurisdictional claims in published maps and institutional affiliations. 
(c) (i) Open Access This article is licensed under a Creative Commons Attribution 4.0 International License, which permits use, sharing, adaptation, distribution and reproduction in any medium or format, as long as you give appropriate credit to the original author(s) and the source, provide a link to the Creative Commons license, and indicate if changes were made. The images or other third party material in this article are included in the article's Creative Commons license, unless indicated otherwise in a credit line to the material. If material is not included in the article's Creative Commons license and your intended use is not permitted by statutory regulation or exceeds the permitted use, you will need to obtain permission directly from the copyright holder. To view a copy of this license, visit http://creativecommons.org/ licenses/by/4.0/.

(C) The Author(s) 2021 